\title{
Hele-Shaw rheometry
}

\author{
Sita Drost ${ }^{\text {a) }}$ \\ Teijin Aramid B.V., P. O. Box 5153, 6802 ED Arnhem, The Netherlands \\ and Laboratory for Aero- and Hydrodynamics, Delft University of Technology, \\ Mekelweg 2, 2628 CD Delft, The Netherlands
}

\author{
Jerry Westerweel \\ Laboratory for Aero- and Hydrodynamics, Delft University of Technology, \\ Mekelweg 2, 2628 CD Delft, The Netherlands
}

(Received 13 June 2013; final revision received 12 September 2013; published 18 October 2013)

\begin{abstract}
Synopsis
In this paper, we describe a novel approach to determine the flow behavior index of a power-law fluid by means of a microfluidic device. The concept of this method is based on a mathematical analysis by Aronsson and Janfalk [Eur. J. Appl. Math. 3, 343-366 (1992)] of Hele-Shaw flow of power-law liquids. We implement this approach by driving a non-Newtonian fluid through a glass microfluidic chip with a 100:1 contraction. The flow in this chip satisfies the Hele-Shaw flow conditions in most of the device. Two conjugate $p$-Laplace equations describe the pressure and stream function in such flows. These equations depend on the flow behavior index, $n$. Therefore, by fitting the $p$-Laplace equation to the velocity field obtained from a micro particle image velocimetry measurement of the flow, the flow behavior index of the fluid in the chip can be determined. Because in practice, fluids rarely show perfectly inelastic power-law behavior, conditions under which the assumption of inelastic flow is valid were derived by analyzing Hele-Shaw flow of an Oldroyd-B fluid. The concept was tested using three different classes of model fluids, a Newtonian fluid, an inelastic power-law fluid, and a Boger fluid. In all three cases, satisfactory results were obtained, with values of $n$ deviating at most $4 \%$ from values measured using conventional rheometry. The method presented here is expected to be potentially useful in online quality control in, for example, polymer or food processing. (C) 2013 The Society of Rheology. [http://dx.doi.org/10.1122/1.4824856]
\end{abstract}

\section{INTRODUCTION}

Contraction flows of non-Newtonian fluids have been a subject of study since the 1960s. This type of flow is relevant for many industrial polymer processing applications, and at high flow rates, it displays a range of interesting dynamics not seen in Newtonian contraction flows and still not completely understood. Comprehensive reviews of the research in this field are, for example, the ones by Boger (1987), White et al. (1987), and Owens and Phillips (2002).

In recent years, the focus of the study of non-Newtonian flow in contractions and other complex geometries has shifted toward microscale geometries. The main advantage of

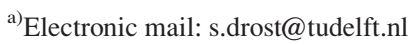


such geometries is that they allow for flows with considerable elastic effects (high Deborah, or Weissenberg, number) combined with negligible influence of inertia (low Reynolds number).

Because of this combination of high deformation rate and low Reynolds number, microfluidic devices also offer new possibilities for rheometry. An additional advantage for rheometry purposes is the low sample volume required in a microfluidic device. An overview of development in microfluidic rheometry up to 2009 was given by Pipe and McKinley (2009). More recent work in this field was done, for example, by Zimmerman and co-workers: Bandalusena et al. (2010) and Craven et al. (2010).

The dimensions of microfluidic devices often allow for fluid mechanical simplifications, for example, Hele-Shaw flow (see also Sec. II A). The Hele-Shaw flow approximation is valid for creeping flows with in-plane dimensions much greater than the depth of the flow. For Newtonian fluids, paradoxically, such-very low Reynolds number-flows behave as inviscid, irrotational potential flow, that is, flow at infinite Reynolds number. Therefore, Hele-Shaw flow is particularly suitable to visualize streamlines of flow around an obstacle. In earlier times, this was used in aerodynamics, for example, in the design of aerofoils. Furthermore, as the governing equations for Hele-Shaw flow are identical to those of flow in a porous medium (Darcy's law), Hele-Shaw flow is also a useful tool to visualize flow in (twodimensional) porous media.

In case of a non-Newtonian fluid, Hele-Shaw flow becomes more complex. In the context of cementing oil wells, Frigaard and co-workers studied Hele-Shaw flow of a viscoplastic, Herschel-Bulkley material, see, for example, Bittleston et al. (2002).

For inelastic power-law fluids, the implications of non-Newtonian behavior for the classical Hele-Shaw flow approximation were mathematically analyzed by Aronsson and Janfalk (1992). ${ }^{1}$ The authors give analytical solutions for a number of special cases and show that the streamline pattern around an obstacle depends on the flow behavior index, or power-law index, $n$.

This dependence of the streamline pattern on the power law index means that it should be possible to determine the rate of shear-thinning or -thickening of a power-law fluid by measuring the shape of its streamlines around an obstacle in a Hele-Shaw cell. As many polymeric liquids show power-law viscosity behavior, at least for a certain range of shear rates, the flow behavior index is a very relevant property.

However, inelastic power-law fluids are very rare in practice. Moreover, as already mentioned, microfluidic devices tend to give a combination of low Reynolds number and high Weissenberg number by design. Therefore, it is important to also investigate elastic effects in Hele-Shaw flow.

In this paper, we investigate how the flow behavior index of a power-law fluid can be determined from the streamline pattern in Hele-Shaw flow around an obstacle. After briefly summarizing the results of Aronsson and Janfalk in Sec. II B, we derive conditions under which their analysis for an inelastic power-law fluid is valid for a viscoelastic fluid (Sec. II C). Subsequently, in Sec. III, we show how the concept was tested experimentally, using a Newtonian liquid, an inelastic power-law fluid, and a Boger fluid. The results are discussed in Sec. IV and the conclusions and recommendations for further study are summarized in Sec. V.

\footnotetext{
${ }^{1}$ From several references, a. o. in Barenblatt's book on theory of fluid flow through natural rocks, Barenblatt et al. (1990), it appears that Entov and co-workers studied the same problem already in the 1970's, in the context of non-linear filtering.
} 


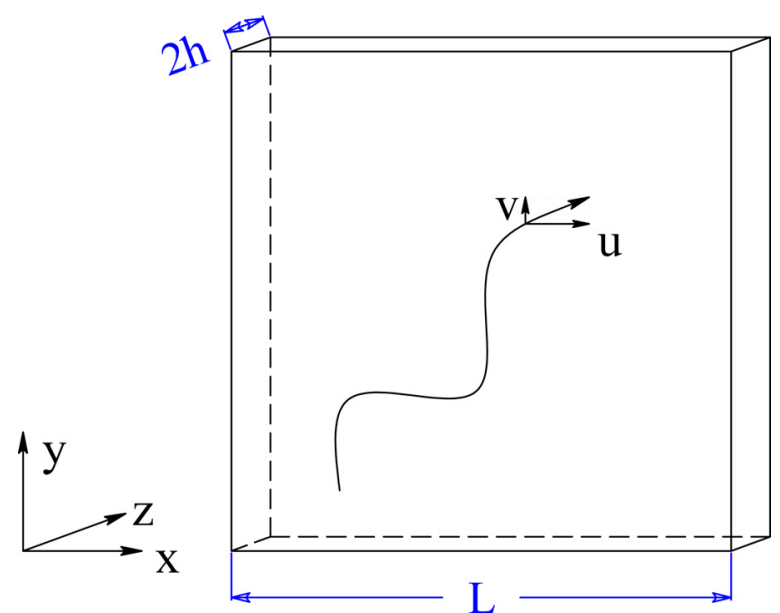

FIG. 1. Geometry of Hele-Shaw flow, the in-plane dimensions are much larger than the depth of the flow, i.e., $L \gg h$.

\section{MATHEMATICAL ANALYSIS}

\section{A. Hele-Shaw flow approximation}

The Hele-Shaw flow approximation is used for an incompressible, creeping flow between two closely spaced parallel plates for which the in-plane dimensions are much larger than the gap width (Fig. 1). This leads to the following assumptions [see, for example, Batchelor (2000) or Milne-Thomson (1996)]:

1. Inertia and body forces are negligible.

2. The velocity normal to the plates is zero, i.e., $w=0$.

3. Of the velocity components in $x$ - and $y$-directions, $u$ and $v$, respectively, the in-plane derivatives are negligible in comparison with the derivatives in $z$-direction: $u_{x}, u_{y}, v_{x}$, $v_{y} \ll u_{z}, v_{z}$. With these assumptions, the momentum equation reduces to $\nabla p=\nabla \cdot \tau$, with $p$ the pressure and $\tau$ the deviatoric stress. The velocity gradient tensor, $\nabla \mathbf{u}$, and the rate of deformation tensor, $\mathbf{D}$, become

$$
\nabla \mathbf{u}=\left[\begin{array}{ccc}
0 & 0 & 0 \\
0 & 0 & 0 \\
u_{z} & v_{z} & 0
\end{array}\right], \quad \mathbf{D}=\frac{1}{2}\left(\nabla \mathbf{u}+(\nabla \mathbf{u})^{T}\right)=\frac{1}{2}\left[\begin{array}{ccc}
0 & 0 & u_{z} \\
0 & 0 & v_{z} \\
u_{z} & v_{z} & 0
\end{array}\right]
$$

respectively.

The classical Hele-Shaw analysis of a Newtonian fluid results in inviscid, irrotational potential flow, with gap-averaged velocity

$$
\overline{\mathbf{u}}=-\frac{h^{2}}{3 \eta} \nabla_{*} p .
$$

Here, $\eta$ is the fluid viscosity and $h$ is the half gap width, as shown in Fig. 1. The equations for the pressure and the stream function in this case are

$$
\begin{array}{ccc}
\frac{\partial^{2} p}{\partial x^{2}}+\frac{\partial^{2} p}{\partial y^{2}}=0 \quad \text { or } \quad \nabla_{*}^{2} p=0, \\
\frac{\partial^{2} \psi}{\partial x^{2}}+\frac{\partial^{2} \psi}{\partial y^{2}}=0 \quad \text { or } \quad \nabla_{*}^{2} \psi=0,
\end{array}
$$

where $\nabla_{*}$ denotes the two-dimensional nabla operator. 
In an actual flow device, the Hele-Shaw approximation is valid at a distance larger than $h$ from any of the bounding walls parallel to the $z$-axis (Fig. 1). As $h \ll L$, this makes a Hele-Shaw cell very suitable to visualize streamlines of flow around an obstacle.

\section{B. Power-law fluid}

Aronsson and Janfalk (1992) analyzed Hele-Shaw flow of a power-law fluid, which is a specific case of a generalized Newtonian fluid. In a generalized Newtonian fluid, the deviatoric stress is given by

$$
\tau=2 \eta(\dot{\gamma}) \mathbf{D}
$$

that is, the viscosity, $\eta$, is assumed to depend on a scalar measure of the deformation rate, $\dot{\gamma}=(2 \mathbf{D}: \mathbf{D})^{\frac{1}{2}}$, which is the second invariant of the rate-of-deformation tensor, D. For a power-law fluid, this dependency is given by

$$
\eta(\dot{\gamma})=K \dot{\gamma}^{n-1}
$$

where the consistency index, $K$, and the flow behavior index, or power-law index, $n$, are positive constants.

Aronsson and Janfalk showed that in Hele-Shaw flow of a power-law fluid, the equations for the pressure and the stream function are so-called $p$-Laplace functions

$$
\begin{gathered}
\frac{\partial}{\partial x}\left(\left|\nabla_{*} p\right|^{\frac{1}{n}-1} \frac{\partial p}{\partial x}\right)+\frac{\partial}{\partial y}\left(\left|\nabla_{*} p\right|^{\frac{1}{n}-1} \frac{\partial p}{\partial y}\right)=0, \\
\frac{\partial}{\partial x}\left(\left|\nabla_{*} \psi\right|^{n-1} \frac{\partial \psi}{\partial x}\right)+\frac{\partial}{\partial y}\left(\left|\nabla_{*} \psi\right|^{n-1} \frac{\partial \psi}{\partial y}\right)=0,
\end{gathered}
$$

or, more compactly,

$$
\begin{aligned}
& \nabla_{*} \cdot\left(\left|\nabla_{*} p\right|^{\frac{1}{n}-1} \nabla_{*} p\right)=0, \\
& \nabla_{*} \cdot\left(\left|\nabla_{*} \psi\right|^{n-1} \nabla_{*} \psi\right)=0 .
\end{aligned}
$$

The depth averaged velocity satisfies

$$
\overline{\mathbf{u}}=-\frac{2 n}{2 n+1}\left(\frac{\nabla_{*} p}{K}\right)^{\frac{1}{n}} h^{\frac{1}{n}+1} .
$$

For $n=1$, these equations simplify to the Laplace equations for Newtonian HeleShaw flow [Eqs. (2) and (3)]. Equations of the $p$-Laplace type are also encountered, for example, in notch and crack growth problems [Atkinson and Champion (1984)] and in nonlinear filtration [Barenblatt et al. (1990)].

The only material property appearing in the equations for the pressure and stream function (5) is the power-law index, $n$. As the stream function is directly related to the flow velocity, this suggests that it should be possible to experimentally determine the value of $n$ of an unknown inelastic power-law fluid from velocity field measurements in Hele-Shaw flow around an obstacle. An obstacle is required to attain a gradient in $p$ and 
$\psi$ [e.g., uniform, unidirectional flow is a trivial solution, which satisfies Eq. (5) for any value of $n$ ]. This idea will be illustrated with the following example.

\section{Illustrative example}

For simple flow geometries, the Laplace equations for Newtonian potential flow can be solved analytically. Analogously, several classes of exact solutions to the $p$-Laplace equations can be derived. In their paper, Aronsson and Janfalk (1992) use hodograph mapping (Legendre transformation) to derive some of these solution classes. They show that classical Newtonian potential flow solutions, such as corner flow and a source doublet [see, for example, Milne-Thomson (1996)], have power-law counterparts with similar streamline patterns, but deviating streamline curvature.

As an illustration, the streamlines of flow in a $90^{\circ}$ salient corner, following from one of the exact solutions given by Aronsson and Janfalk, are plotted in Fig. 2 for different values of $n$. It can be clearly seen that with increasing $n$, the streamlines tend to follow the walls more closely.

This may seem counter-intuitive at first sight, as we are used to parabolic velocity profiles in pipe and channel flow becoming more blunt with decreasing value of $n$. However, in the case of Hele-Shaw flow, the wall shear stress at the top and bottom plates is dominant and the flow behavior in the $x-y$ plane is effectively inviscid. This can also be seen in the exact solutions [Aronsson and Janfalk (1992)], where the flow velocity at the walls is nonzero (in practice, the Hele-Shaw approximation ceases to hold when the distance to the wall becomes smaller than $h$ ). To get a better feeling for what happens here, we look at the simple case of a unidirectional flow, where the shear stress on the top and bottom walls of the Hele-Shaw cell is given by

$$
\tau_{w}=K\left(\frac{2 n+1}{2 n} \frac{\bar{u}}{h}\right)^{n} .
$$

This expression shows that the increase of $\tau_{w}$ with $\bar{u}$ is faster than linear for $n>1$ and slower than linear for $n<1$. Because the flow velocity increases with distance from the corner, it seems plausible that a shear-thickening fluid follows the corner contour more closely.
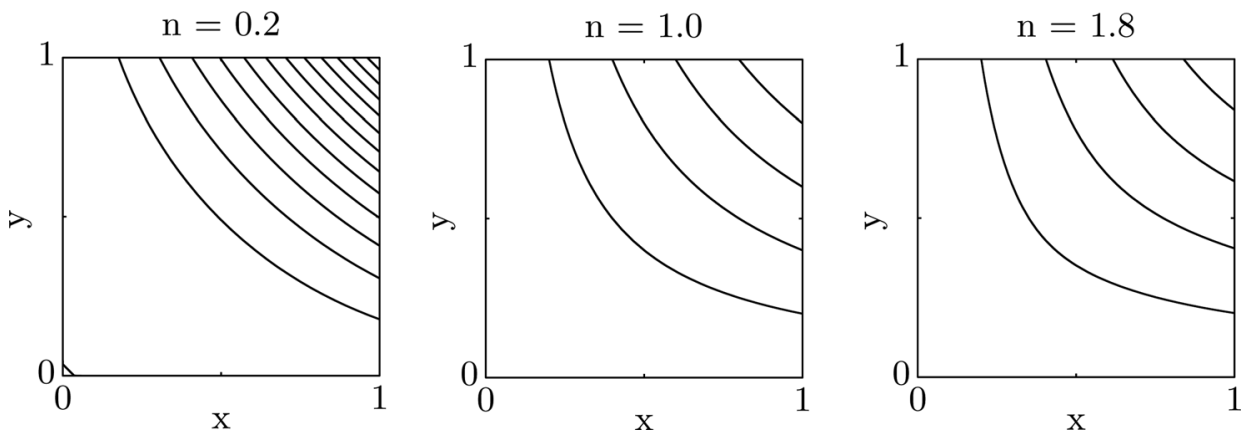

FIG. 2. Streamlines, i.e., lines of constant $\psi$, of flow in a $90^{\circ}$ salient corner, following from an exact solution to the 2D $p$-Laplace equations, Eq. (5), units on the axes are arbitrary. The positive $x$ - and $y$-axes represent (semiinfinite) solid walls, the flow is from top left to bottom right. Left: $n=0.2$ (shear-thinning), middle: $n=1$ (Newtonian), right: $n=1.8$ (shear-thickening). 
As the example with the exact solutions in Fig. 2 shows, the streamline curvature of Hele-Shaw flow past an obstacle changes as a function of $n$. Therefore, it should be possible to experimentally determine the value of $n$ of an unknown inelastic power-law fluid by measuring the streamlines or-directly related - the velocity field near an obstacle.

Before testing this approach in practice, we first analyze the implications of viscoelastic behavior in Hele-Shaw flow, as actual non-Newtonian fluids are rarely perfectly inelastic when they show power-law behavior.

\section{Viscoelastic effects}

We expect that the inelastic power-law approximation will be valid for viscoelastic fluids as well, as long as viscous effects are dominant in the flow, that is, at low Weissenberg number. To determine under which conditions elastic effects will be negligible, we study steady Hele-Shaw flow of an Oldroyd-B fluid. The dimensionless momentum equation in this case is

$$
\begin{gathered}
\frac{\eta_{0}}{\eta_{p}} \nabla p=\frac{\eta_{s}}{\eta_{p}} \nabla^{2} \mathbf{u}+\frac{1}{W i} \nabla \cdot \mathbf{A}, \\
\mathbf{A}=\mathbf{I}-W i \stackrel{\nabla}{\mathbf{A}}=\mathbf{I}-W i\left[(\mathbf{u} \cdot \nabla) \mathbf{A}-\mathbf{A} \cdot \nabla \mathbf{u}-(\nabla \mathbf{u})^{\mathrm{T}} \cdot \mathbf{A}\right] .
\end{gathered}
$$

Here, $\mathbf{A}$ is the polymer stretch tensor, with $\mathbf{A}$ its upper convected derivative, $\eta_{s}$ and $\eta_{p}$ are the solvent and polymer viscosity (i.e., added viscosity due to the presence of polymer), respectively, and $\eta_{0}=\eta_{s}+\eta_{p}$. Velocity and pressure are scaled as $u^{*}=u / U$ and $p^{*}=h p / \eta_{0} U$, respectively, where the asterisks indicate the dimensionless quantities [following convention, these asterisks have been omitted in Eq. (6)]. Finally, Wi is the Weissenberg number, $W i=\lambda U / h$, with $\lambda$ the relaxation time of the fluid.

A weak flow expansion is used to study the first effects of elasticity, at low Wi. Expanding $\mathbf{u}, p$, and $\mathbf{A}$ in terms of $W i$,

$$
\begin{array}{r}
\mathbf{u}=\mathbf{u}_{0}+W i \mathbf{u}_{1}+\ldots, \\
p=p_{0}+W i p_{1}+\ldots \\
\mathbf{A}=\mathbf{A}_{0}+W i \mathbf{A}_{1}+\ldots
\end{array}
$$

and collecting terms with equal powers of $W i$ gives for $\mathbf{A}$

$$
\begin{gathered}
\mathbf{A}_{0}=\mathbf{I}, \\
\mathbf{A}_{i+1}=-\overrightarrow{\mathbf{A}}_{i} .
\end{gathered}
$$

The momentum equations of order $W i^{0}, W i^{1}$, and $W i^{2}$ then become

$$
\begin{aligned}
W i^{0}: 0 & =\nabla \cdot \mathbf{I}, \\
W i^{1}: \frac{\eta_{0}}{\eta_{p}} \nabla p_{0} & =\frac{\eta_{s}}{\eta_{p}} \nabla^{2} \mathbf{u}_{0}+\nabla^{2} \mathbf{u}_{0}=\frac{\eta_{0}}{\eta_{p}} \nabla^{2} \mathbf{u}_{0}, \\
W i^{2}: \frac{\eta_{0}}{\eta_{p}} \nabla p_{1} & =\frac{\eta_{s}}{\eta_{p}} \nabla^{2} \mathbf{u}_{1}+2 \stackrel{\nabla}{\mathbf{D}} .
\end{aligned}
$$

The zeroth-order equation simply implies no flow, and the first-order equation gives Newtonian flow with an added viscosity term due to the polymer. After applying the Hele-Shaw assumptions, the components of the second-order equation become 


$$
\begin{gathered}
\frac{\eta_{0}}{\eta_{p}} \frac{\partial p_{1}}{\partial x}=\frac{\partial}{\partial z}\left(\frac{\eta_{s}}{\eta_{p}} \frac{\partial u_{1}}{\partial z}-\frac{\partial^{2} u_{0}}{\partial z \partial x} u_{1}-\frac{\partial^{2} u_{0}}{\partial z \partial y} v_{1}\right), \\
\frac{\eta_{0}}{\eta_{p_{1}}} \frac{\partial p}{\partial y}=\frac{\partial}{\partial z}\left(\frac{\eta_{s}}{\eta_{p_{1}}} \frac{\partial v_{1}}{\partial z}-\frac{\partial^{2} v_{0}}{\partial z \partial x} u_{1}-\frac{\partial^{2} v_{0}}{\partial z \partial y} v_{1}\right), \\
\frac{\eta_{0}}{\eta_{p_{1}}} \frac{\partial p_{1}}{\partial z}=0 .
\end{gathered}
$$

From the solution of the Newtonian case, we know that $u_{0}, u_{0, x}, u_{0, y}=\mathcal{O}\left(h^{2}\right), u_{0, z}$ $=\mathcal{O}(h)$ and similar for $v_{0}$. If we assume this to hold for $u_{1}$ too, the last two terms on the right-hand side of Eqs. (8a) and (8b) can be neglected, and the flow field of an Oldroyd-B fluid, up to second order in $W i$, will be the same as for a Newtonian liquid.

This result is similar to the planar flow theorem of Tanner and Pipkin [Tanner (1966)], which states that given a velocity field that satisfies the equations for creeping plane flow of an incompressible Newtonian fluid, the same velocity field also satisfies the equations for creeping plane flow of an incompressible second-order fluid. However, in our case, the flow is not truly planar, and hence, it should satisfy the additional condition that the depth-based Weissenberg number is sufficiently small, $W i \ll 1$.

To determine a more definitive critical value for $W i$, and to see how elastic effects influence the streamline pattern, a more extensive study is required, for example, experimentally or numerically. Unfortunately, this is beyond the scope of the current paper.

\section{Combination of power-law and viscoelastic behavior}

The situation becomes more complex in case of a fluid that shows both sheardependent viscosity and viscoelastic behavior. For Hele-Shaw flow of a JohnsonSegalman-Oldroyd model, Fast et al. (2001) derived a generalized Darcy's law

$$
\overline{\mathbf{u}}=-\frac{\nabla_{*} p}{\bar{\mu}},
$$

where $\overline{\mathbf{u}}$ is again the gap-averaged in-plane velocity, $\nabla *$ indicates the two-dimensional nabla-operator, and $\bar{\mu}=\bar{\mu}\left(W i^{2}\left|\mathbf{u}_{z}\right|^{2}\right)$ is introduced as an effective viscosity.

This generalized Darcy's law is the result of the leading order equations of an asymptotic expansion in the depth-to-width ratio, $\epsilon=h / L$. This means that, even to zeroth order in $\epsilon$, the effective viscosity is a function of both the Weissenberg number and the shear rate. However, as long as $W i \ll 1$, its influence on the effective viscosity is negligible, and it is expected that determination of the power-law index from a measured velocity field should still be possible.

\section{EXPERIMENTAL}

From the analysis in Sec. II, it follows that it should be possible to determine the power-law index of a power-law fluid from the shape of the streamlines in Hele-Shaw flow around an obstacle. Effects of elasticity are negligible as long as the Weissenberg number is kept sufficiently low, $W i \ll 1$.

As a first test of the validity of this approach, we recorded flow fields in a 100:1 microfluidic contraction. The dimensions of this flow cell meet the criteria for a Hele-Shaw cell, except close to the contraction, and the salient corners are suitable to serve as "obstacles." As test fluids, three classes of fluids were used: a Newtonian fluid, an 
inelastic power-law fluid, and a Boger fluid. The velocity fields were measured using micro particle image velocimetry [ $\mu$ PIV, Adrian and Westerweel (2011)].

\section{A. Measurement setup}

For the experiments, a custom-made glass flow cell with a 100:1 contraction and free outflow (Micronit Microfluidics) was used. The dimensions of this flow cell are shown in Fig. 3 (left). The flow cell was clamped vertically in a holder and fed by a rate-controlled syringe pump (KD Scientific). Because it is a Hele-Shaw flow, the flow is fully developed almost immediately after entering the cell. Test measurements were taken, which confirmed that the velocity profile in the flow cell is uniform, up to the part where the flow starts to accelerate toward the contraction.

The flow was seeded with $2 \mu \mathrm{m}$ tracer particles and illuminated from behind by a pulsed light-emitting diode (LED). A double-frame PIV camera (LaVision Imager Intense) with a $105 \mathrm{~mm}$ focal length lens (Nikon) was used to record image pairs of the flow at a rate of $5 \mathrm{~Hz}$, with a delay of $\Delta \mathrm{t}=500 \mu$ s between the frames in an image pair and an exposure time of $10 \mu \mathrm{s}$ for each frame. The velocity field in the slower parts of the flow was determined using only the first frame of each image pair, while for the faster part of the flow, near the outlet (where the Hele-Shaw approximation ceases to hold), both frames were needed. The complete setup is depicted schematically in Fig. 3 (right).

\section{B. Method of determining $n$}

To determine the value of the power-law index, we chose to use velocity field measurements. Under the conditions for inelastic power-law Hele-Shaw flow, the stream function satisfies Eq. (4b). This equation can be rewritten in terms of velocity and velocity gradients, which can be measured using $\mu \mathrm{PIV}$

$$
(n-1)\left(u^{2}+v^{2}\right)^{n-3 / 2}\left(v^{2} \frac{\partial v}{\partial x}-2 u v \frac{\partial v}{\partial y}-u^{2} \frac{\partial u}{\partial y}\right)+\left(u^{2}+v^{2}\right)^{n-1 / 2}\left(\frac{\partial v}{\partial x}-\frac{\partial u}{\partial y}\right)=0 .
$$

The power-law index of the tested fluid is that value of $n$ for which the measured velocity field best satisfies this equation, that is, for which the absolute value of the left-hand side of the equation is minimal.
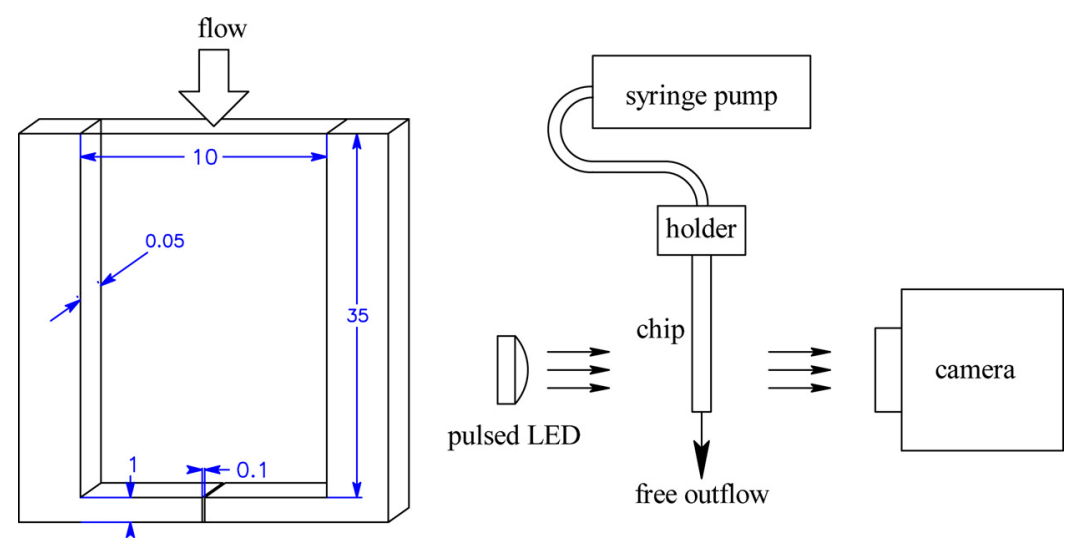

FIG. 3. Left: Dimensions of the flow cell with 100:1 contraction (not to scale, dimensions in mm). Right: Schematic drawing of measurement setup. 
Because PIV is a whole-field method that yields both in-plane velocity components and their spatial derivatives in a discrete number of grid points, Eq. (10) can be evaluated in each grid point. An error norm can then be defined, which is to be minimized to find the value of $n$.

The magnitude of the velocity is not important here, because the value of $n$ is related to the shape of the streamlines and these are independent of the $z$-position in the HeleShaw cell. Therefore, it is not necessary to know whether the used $\mu$ PIV setup results in measuring the average velocity, or the maximum velocity, or some fraction of the maximum velocity [cf. Kloosterman et al. (2011)]. To still give an idea, according to a paper by Olsen and Adrian (2000), the lens that was used in our setup results in measuring the velocity averaged over a depth of around $50 \mu \mathrm{m}$, which is in our case the depth of the entire cell.

Before applying this approach to actual measurement data, it was first tested on synthetic data. The matLAB function fminsearch was used for the minimization procedure; this is a nonlinear unconstrained optimization function (for more information, the reader is referred to the website of The Mathworks). We used the sum of the absolute errors [i.e., the absolute value of the left-hand side of Eq. (10) in each grid point] as a norm for minimization and $n$ as the fitting parameter. Adding Gaussian white noise to an analytical solution showed that, provided a reasonable initial guess was provided, the fitting procedure converged to a result with less than $5 \%$ error, up to a signal-to-noise ratio of $15 \mathrm{~dB}$, which was considered satisfactory for the present purpose.

This minimum signal-to-noise ratio is also something to keep in mind when using actual PIV data. A minimum signal-to-noise ratio of $15 \mathrm{~dB}$ means that the amplitude of the PIV correlation peak should be at least $\sqrt{10^{1.5}} \approx 5.6$ times larger than other, noiseinduced, peaks in an interrogation window [Adrian and Westerweel (2011)].

In our PIV measurements, we used the correlation average over 100 images, for optimal accuracy [Adrian and Westerweel (2011)]. The images were first analyzed using $128 \times 128$ pixel windows, with $50 \%$ overlap. The resulting velocity field was subsequently used as input for a finer analysis, with $64 \times 64$ pixel windows, $50 \%$ overlap, and window shifting. Only the part of the velocity field where the Hele-Shaw approximation is valid was used for fitting, that is, the part where the in-plane velocity gradients are much smaller than those in the depth-direction (i.e., sufficiently far from the contraction and the walls). This resulted in velocity fields of approximately $15 \times 25$ grid points, as shown in Fig. 4.

\section{Test fluids}

As a Newtonian test fluid, glycerol (Boom, technical grade) was used. To approach inelastic power-law behavior as closely as possible, a $0.02 \mathrm{wt}$. \% xanthan gum solution in glycerol-water (75-25 wt. \%, xanthan gum from Sigma-Aldrich) was used. According to Rodrigo Vélez-Cordero et al. (2011) and Rodrigo Vélez-Cordero and Zenit (2011), this solution shows negligible elastic behavior and is shear-thinning with $n=0.85$ in the measured range of $10^{-1} \mathrm{~s}^{-1} \leq \dot{\gamma} \leq 10^{3} \mathrm{~s}^{-1}$. With higher xanthan gum concentrations, lower values of $n$ can be attained. We chose a relatively low concentration, however, in order to minimize elastic effects. The Boger fluid of choice for the experiments was a solution of $30 \mathrm{wt}$. \% polyethyleneglycol (PEG), $8000 \mathrm{~g} / \mathrm{mol}$. and $0.06 \mathrm{wt} \%$ polyethyleneoxide (PEO), $4 \times 10^{6} \mathrm{~g} / \mathrm{mol}$. (both from Sigma-Aldrich) in water, as was also used by, among others, Dontula et al. (1998) and Sankaran et al. (2013).

The rheological properties of the PEG-PEO and xanthan gum solutions were measured on a TA Ares-G2 rheometer with a Couette geometry and a gap width of $5 \mathrm{~mm}$. 

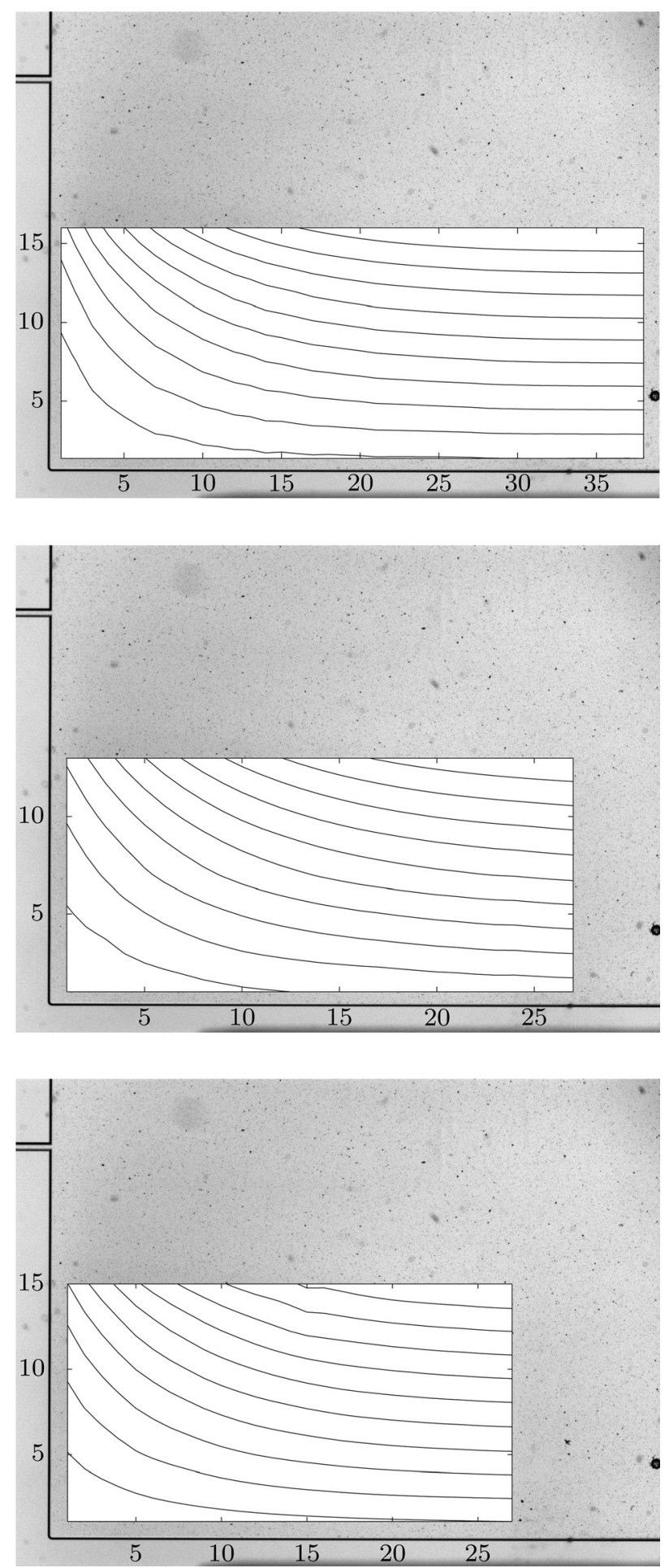

FIG. 4. Streamlines resulting from PIV measurements, superimposed on an image of the Hele-Shaw cell (flow is from right to left). Top: Glycerol, $20 \mu \mathrm{l} / \mathrm{min}$, middle: Xanthan gum solution, $20 \mu \mathrm{l} / \mathrm{min}$, bottom: PEG-PEO Boger fluid, $5 \mu \mathrm{l} / \mathrm{min}$. The part of the flow where the streamlines are shown is also the part that was used for the fitting procedure. The axes represent the PIV interrogation window number. 

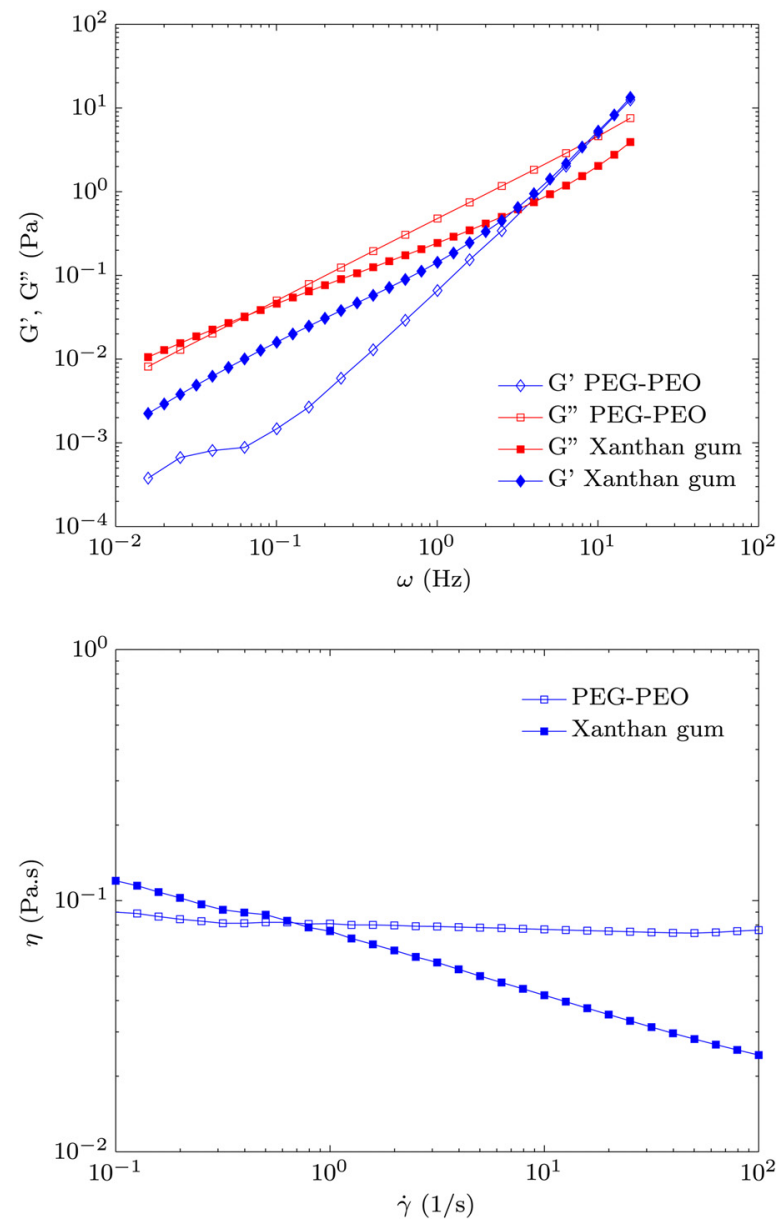

FIG. 5. Rheological behavior of the xanthan gum and PEG-PEO solutions used as test fluid. Top: Loss and storage modulus in an oscillatory frequency sweep; bottom: viscosity in steady shear.

Oscillatory as well as steady measurements were performed. The results are shown in Fig. 5. The glycerol was considered to be Newtonian, with $n=1$.

Our xanthan gum solution shows shear-thinning with $n=0.86$, which agrees sufficiently with the value measured by Rodrigo Vélez-Cordero et al. However, the crossover of $G^{\prime}$ and $G^{\prime \prime}$ in the oscillatory measurements occurs at a frequency that is about a decade lower than reported by Rodrigo Vélez-Cordero et al. This makes the assumption of negligible elasticity rather questionable. Identifying the cause of this difference in measured rheological behavior is beyond the scope of this paper. Also more extensive measurements, or curve-fits, to determine a relaxation time (spectrum) were not considered here.

\section{RESULTS AND DISCUSSION}

The velocity fields in glycerol and in the xanthan gum solution were measured at a flow rate of $20 \mu \mathrm{l} / \mathrm{min}$. For the Boger fluid, the Zimm relaxation time [Zimm (1956)] of $4.2 \mathrm{~ms}$ was assumed, as calculated by Sankaran et al. (2013). A flow rate of $20 \mu \mathrm{l} / \mathrm{min}$ would then result in a depth-based Weissenberg number, $\lambda U / h$, of $0.11\left(\dot{\gamma} \approx 25 \mathrm{~s}^{-1}\right)$. This 
is expected to be low enough to not see any elastic effects, but to be on the safe side, a flow rate of $5 \mu \mathrm{l} / \mathrm{min}$ was used, giving $W i=0.03\left(\dot{\gamma} \approx 7 \mathrm{~s}^{-1}\right)$.

A flow rate of $20 \mu \mathrm{l} / \mathrm{min}$ yields an average shear rate of $\partial u / \partial z \approx 25 \mathrm{~s}^{-1}$ in our flow cell, which is well in the shear-thinning regime of our xanthan gum solution. At $5 \mu \mathrm{l} / \mathrm{min}$, the average shear rate is approximately $7 \mathrm{~s}^{-1}$ at which the PEG-PEO Boger fluid shows constant viscosity behavior (see Fig. 5).

The recording of 100 image pairs took about half a minute, meaning that approximately $10 \mu \mathrm{l}$ of fluid was needed for the xanthan gum and glycerol measurements, and $2.5 \mu \mathrm{l}$ for the Boger fluid measurement.

The measured streamlines are shown in Fig. 4, superimposed on an image of the flow cell. The data used for the generation of these streamlines are also the data that were used in the fitting procedure. The values of $n$ obtained by fitting equation (10) to the measured velocity fields are given in Table I, together with the expected values, the relative error, and an indication of the PIV data quality, $S$.

In all three cases, the resulting values of $n$ differ less than 5\% from the values measured with conventional rheometry, even in the case of the PEG-PEO Boger fluid, where the PIV data quality was a bit lower than the critical value determined in Sec. III B. The resulting value of $n$ depends slightly on the part of the flow field that is used for the fit, but in our measurements, this dependency was only visible in the third decimal of $n$.

Additional measurements are necessary to further asses the sensitivity of the fitting procedure to the choice of the data set. Also the effect of elasticity should be studied more extensively; experiments with a fluid with known relaxation time should be carried out at different flow rates to see how the streamlines change with increasing Weissenberg number and to determine a more definite critical value of $W i$.

For our tests, we used flow cells with a contraction geometry, but in principle, any Hele-Shaw flow with curved streamlines can be used. For example, Hele-Shaw flow around a cylinder might be even better suitable for this purpose, because in that geometry the Hele-Shaw approximation would be valid throughout the complete flow cell

Because in practice non-Newtonian fluids are virtually never perfectly inelastic, information about the elastic behavior of a fluid is needed a priori, in order to choose the flow settings such that the inelastic approximation may be used. Also the shear rate range in which the fluid shows shear-thinning — or thickening_behavior should roughly be known. Furthermore, the fit gives only the power-law index of a fluid, instead of the complete viscosity curve, making the use of additional measurements (e.g., pressure drop at a number of flow rates) necessary if also the consistency index of the test fluid is required. Therefore, our method does not seem useful as a replacement of conventional rheometry methods.

However, because of its simplicity, our method could be a useful tool for (online) quality control in processes involving non-Newtonian flow, like polymer or food processing. Contrary to conventional rheometry, our method can be operated at a single flow rate. Therefore, the time required for one measurement is relatively short (ca. $30 \mathrm{~s}$ in our

TABLE I. Results of streamline fitting, $n_{\mathrm{fit}}$ is the power law index resulting from the fitting procedure, $n_{\mathrm{rheom}}$ is the value resulting from conventional rheometry on the samples, $S$ is a measure of the signal-to-noise ratio of the correlation peak, with "av" indicating the average value and "crit" the percentage of the measurement points with a signal-to-noise ratio larger than the critical value of 5.6 (see Sec. III B).

\begin{tabular}{lllccc}
\hline \hline & $n_{\text {fit }}$ & $n_{\text {rheom }}$ & Error $(\%)$ & $S_{\text {av }}$ & $S \geq S_{\text {crit }}(\%)$ \\
\hline Glycerol & 0.999 & 1.00 & 0 & 29 & 99 \\
Xanthan gum & 0.868 & 0.860 & 1 & 22 & 89 \\
PEG-PEO & 1.04 & 1.00 & 4 & 5.1 & 12 \\
\hline \hline
\end{tabular}




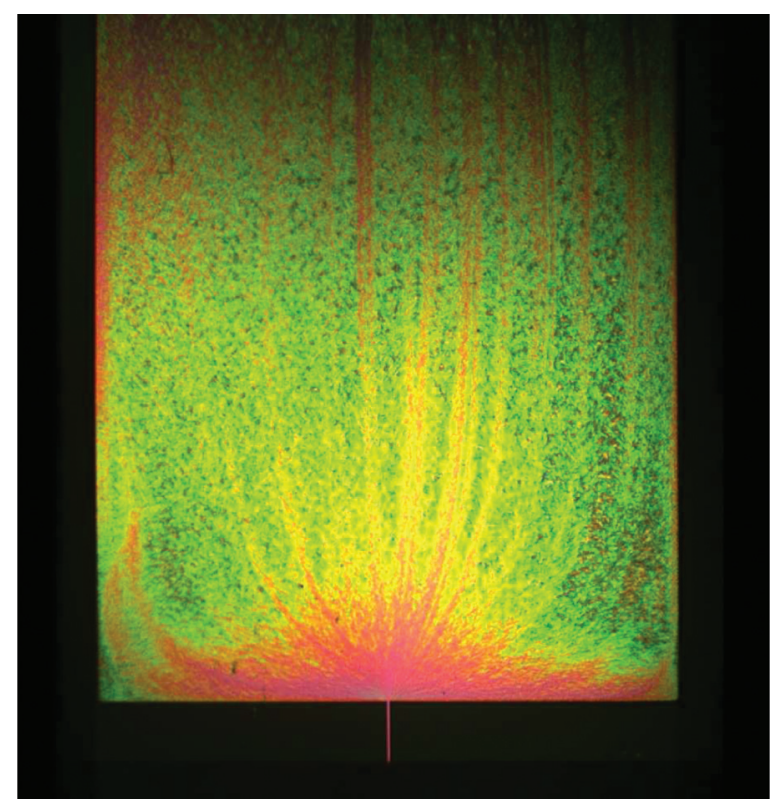

FIG. 6. Contraction flow of a liquid crystalline solution of $10 \mathrm{wt}$ \% of poly-2,2'-disulfonylbenzidine-terephtalamide (PBDT) in water (sample obtained from J. Gao, TU Delft). The same flow geometry was used as in the other experiments in this paper. It was placed between two oppositely oriented circular polarizers to improve the visibility of the structure in the flow. No tracer particles are needed here to measure the velocity field and/or the streamlines.

experiments). Processing of the measured data may take some more time. However, because the value of $n$ is linked to the shape of the streamlines, after calibration, measurements should be possible using image analysis instead of PIV.

In case of optically anisotropic fluids, like liquid crystalline fluids (Fig. 6), both PIV and image analysis can be done without any additions to the flow, whereas for other fluids, the injection of tracer particles would be necessary. Because the sample flow rate required for a measurement is very small $[\mathcal{O}(10) \mu \mathrm{l} / \mathrm{min}$ in our experiments] compared with the scale of typical industrial processes, the amount of fluid that is polluted with tracer particles is not expected to pose a problem. The costs for flow cells such as the one used for our measurements can be quite low, especially when the cells are produced in large quantities. Therefore, in an industrial application, disposable cells would probably be the cheapest and easiest option.

\section{CONCLUSIONS AND RECOMMENDATIONS}

Tests with three different classes of model fluids showed that it is possible to determine the power-law index of a non-Newtonian fluid from streamline curvature in HeleShaw flow. An equation derived by Aronsson and Janfalk (1992), for Hele-Shaw flow of inelastic power-law fluids, was fitted to velocity fields obtained by PIV, with $n$ as the only fitting parameter. An analysis of viscoelastic effects showed that the inelastic power-law approximation may also be used for a viscoelastic fluid, as long as the depth-based Weissenberg number is much smaller than $1, W i=\lambda U / h \ll 1$. To our best knowledge, this method of determining the power-law index has not been published before. 
Contrary to conventional rheometry, it is not necessary to use a range of different flow rate settings to determine the power-law index of a fluid. This makes the time needed to take a measurement relatively short and the required sample volume relatively low. The time required to process the measurement data may be longer, however.

The setup needed for $\mu$ PIV measurements in our Hele-Shaw cell is comparatively simple. Because of the low flow velocity, an LED backlight can be used, in combination with a standard digital camera at a frame rate of a few Hertz. This is cheaper and easier to implement than a conventional PIV setup, with a pulsed laser and a double-frame PIV camera. Moreover, as only the shape of the streamlines is important for the measurement, it is unnecessary to determine the depth of correlation of the setup (see Sec. III B).

A drawback of our method is that a priori knowledge of the elastic and sheardependent behavior of the sample is required. A reasonable estimate of the relaxation time is needed, because in order for the inelastic power-law assumption to be valid, the depth-based Weissenberg number of the flow should be sufficiently small. At the same time, the characteristic shear rate in the Hele-Shaw cell should fall in the range where the fluid viscosity shows shear rate-dependent behavior. Therefore, our method does not seem suitable as a replacement for conventional rheometry methods. However, its simplicity and low sample volume make our method a good candidate for online quality control, especially if cheap, disposable flow cells are used.

More extensive experimental work is required to determine more definite conditions under which our method may be used. For example, the influence of elasticity on streamline curvature should be investigated, a more definite critical Weissenberg number should be determined, and more specific conditions for choosing a data set for the fitting procedure should be derived (i.e., minimum size, data quality, maximum velocity gradient). Also more attention might be paid to the influence of the choice of a certain error norm on the quality of the fit.

\section{ACKNOWLEDGMENTS}

The authors would like to thank Mr. B. Norder and Dr. R. Delfos of TU Delft and Mrs. Ing. M. H. M. Meeusen of Teijin Aramid for their help with the rheometry of the test fluids, Ing. E. F. J. Overmars of TU Delft for his help with the PIV measurements, Ir. D. A. Dros of Teijin Aramid for his helpful information on the preparation of the Boger fluid, and Teijin Aramid B.V. for their financial support.

\section{References}

Adrian, R. J., and J. Westerweel, Particle Image Velocimetry, Volume 30 of Cambridge Aerospace Series (Cambridge University Press, New York, 2011).

Aronsson, G., and U. Janfalk, "On Hele-Shaw flow of power-law fluids," Eur. J. Appl. Math. 3, 343-366 (1992).

Atkinson, C., and C. R. Champion, "Some boundary-value problems for the equation $\nabla \cdot\left(|\nabla \phi|^{n} \nabla \phi\right)=0$," Q. J. Mech. Appl. Math. 37(3), 401-419 (1984).

Bandalusena, H. C. H., W. B. Zimmerman, and J. M. Rees, "Creeping flow analysis of an integrated microfluidic device for rheometry,” J. Non-Newtonian Fluid Mech. 165, 1302-1308 (2010).

Barenblatt, G. I., V. M. Entov, and V. M. Ryzhik, Theory of Fluid Flows through Natural Rocks (Springer, Dordrecht, The Netherlands, 1990).

Batchelor, G. K., An Introduction to Fluid Dynamics, First Cambridge Mathematical Library edition (Cambridge University Press, Cambridge, UK, 2000). 
Bittleston, S. H., J. Ferguson, and I. A. Frigaard, "Mud removal and cement placement during primary cementing of an oil well," J. Eng. Math. 43, 229-253 (2002).

Boger, D. V., "Viscoelastic flows through contractions," Annu. Rev. Fluid Mech. 19, 157-182 (1987).

Craven, T. J., J. M. Rees, and W. B. Zimmerman, "Pressure sensor positioning in an electrokinetic microrheometer device: Simulations of shear-thinning liquid flows," Microfluid. Nanofluid. 9, 559-571 (2010).

Dontula, P., C. W. Macosko, and L. E. Striven, "Model elastic liquids with water-soluble polymers," AIChE J. 44(6), 1247-1255 (1998).

Fast, P., L. Kondic, M. J. Shelley, and P. Palffy-Muhoray, "Pattern formation in non-Newtonian hele-shaw flow," Phys. Fluids 13(5), 1191-1212 (2001).

Kloosterman, A., C. Poelma, and J. Westerweel, "Flow rate estimation in large depth-of-field micro-piv," Exp. Fluids 50(6), 1587-1599 (2011).

Milne-Thomson, L. M., Theoretical Hydrodynamics (Dover Publications, Mineola, NY, 1996).

Olsen, M. G., and R. G. Adrian, "Out-of-focus effects on particle image visibility and correlation in microscopic particle image velocimetry,” Exp. Fluids 29(1), S166-S174 (2000).

Owens, R. G., and T. N. Phillips, Computational Rheology (Imperial College, London, 2002).

Pipe, C. J., and G. H. McKinley, “Microfluidic rheometry,” Mech. Res. Commun. 36, 110-120 (2009).

Sankaran, A. K., D. A. Dros, J. J. Meerman, M. T. Kreutzer, and S. J. Picken, "Increasing the stability of high contraction ratio flow of boger fluids by pre-deformation," J. Non-Newtonian Fluid Mech. 196, 27-35 (2013).

Tanner, R. I., "Plane creeping flows of incompressible second-order fluids," Phys. Fluids 9, 1246-1247 (1966).

Rodrigo Vélez-Cordero, J., D. Sámano, P. Yue, J. J. Feng, and R. Zenit, "Hydrodynamic interaction between a pair of bubbles ascending in shear-thinning inelastic fluids," J. Non-Newtonian Fluid Mech. 166, 118-132 (2011).

Rodrigo Vélez-Cordero, J., and R. Zenit, "Bubble cluster formation in shear-thinning inelastic bubbly columns," J. Non-Newtonian Fluid Mech. 166(1-2), 32-41 (2011).

White, S. A., A. D. Gotsis, and D. G. Baird, "Review of the entry flow problem: Experimental and numerical," J. Non-Newtonian Fluid Mech. 24(2), 121-160 (1987).

Zimm, B. H., "Dynamics of polymer molecules in dilute solution: Viscoelasticity, flow birefringence and dielectric loss,” J. Chem. Phys. 24(2), 269-278 (1956). 